\title{
Retraction: Exploration of Write-Ahead Logging
}

Haiyu Li

Several conference proceedings have been infiltrated by fake submissions generated by the SCIgen computer program. Due to the fictional content the chapter "Exploration of Write-Ahead Logging" by "Haiyu Li" has been retracted by the publisher. Measures are being taken to avoid similar breaches in the future. 